\title{
La evaluación formativa, ¿Realidad o buenas intenciones? Estudio de caso en docentes del nivel primario
}

\section{Formative Assessment, Reality or Good Intentions? Case study in primary level teachers}

\author{
Yárida Falcón Ccenta \\ Universidad César Vallejo, Perú \\ ORCID: https://orcid.org/0000-0002-5763-7542 \\ Jorge Aguilar - Hernando \\ Universidad Cesar Vallejo, Perú \\ ORCID: https://orcid.org/0000-0002-4652-2774 \\ Carlos Augusto Luy-Montejo \\ Universidad Privada del Norte, Perú \\ ORCID: https://orcid.org/0000-0003-0824-7959 \\ John Morillo-Flores \\ Universidad Privada San Juan Bautista, Perú \\ ORCID: https://orcid.org/0000-0002-2136-4458
}

Received 10-12-20 Revised 11-25-20

Accepted 02-13-21 On line 02-27-21

*Correspondence

Email: Yaridanora@gmail.com
Cite as:

Falcón, Y., Aguilar-Hernando, J., Luy-Montejo, C., \& Morillo-Flores, J. (2021). La evaluación formativa, ¿Realidad o buenas intenciones? Estudio de caso en docentes del nivel primario. Propósitos y Representaciones, 9(1), e1041. Doi: http://dx.doi.org/10.20511/pyr2021.v9n1.1041 


\begin{abstract}
Resumen
Para desarrollar el proceso educativo enmarcado dentro del enfoque por competencias, se requiere implementar cambios en los subprocesos, siendo de interés en este estudio el cambio en la evaluación del aprendizaje por la evaluación para el aprendizaje lo que implica un cambio paradigmático que requiere deconstruir una práctica arraigada en docentes. Se presupone que su implementación exitosa en la EBR dependerá de que los actores educativos interioricen y se concienticen de la necesidad de este cambio en la evaluación, ello generaría predisposición positiva a su aplicación y las capacitaciones, talleres y otras estrategias empleadas para difundirla sean aprovechadas para adquirir el conocimiento en que se respalden las estrategias para una aplicación eficaz. Frente a este fenómeno pedagógico de gran connotación social y ante la renuencia y la ineficacia observada en las escuelas peruanas, resulta conveniente analizar por qué la evaluación formativa difundida y plasmada en los documentos oficiales guías, no es aún una realidad en la práctica docente. Las respuestas se buscaron en un grupo de docentes implicados en su implementación y se contrastaron con las opiniones de quienes monitorean oficialmente su implementación en las aulas, hallándose desconocimiento en los procesos de esta evaluación formativa y una pertinaz resistencia a su aplicación que subyace en los maestros, quienes aún emplean acciones propias de otras formas de evaluar.
\end{abstract}

Palabras clave: evaluación formativa, planificación, implementación, recojo de información, retroalimentación.

\title{
Summary
}

To develop the educational process framed within the competence approach, it is necessary to implement changes in the sub-processes, being of interest in this study the change in the evaluation of learning by the evaluation for learning, which implies a paradigmatic change that requires deconstructing a practice rooted in teachers. It is assumed that its successful implementation in the EBR will depend on the educational actors internalize and become aware of the need for this change in the evaluation, this would generate a positive predisposition to its application and the training, workshops and other strategies used to disseminate it are used to acquire the knowledge to support strategies for effective implementation. Faced with this pedagogical phenomenon of great social connotation and the reluctance and inefficiency observed in Peruvian schools, it is convenient to analyze why the formative evaluation disseminated and embodied in official guide documents is not yet a reality in teaching practice. The answers were sought in a group of teachers involved in its implementation and were contrasted with the opinions of those who officially monitor its implementation in the classrooms, finding ignorance in the processes of this formative evaluation and a stubborn resistance to its application that underlies the teachers, who still use their own actions in other ways of evaluating.

Keywords: Planning, implementation, information gathering, feedback.

Introducción: formative evaluation, planning, implementation, information gathering, feedback.

Las investigaciones de carácter cualitativo sobre evaluación formativa desarrolladas por García y López (2015) determinaron el grado de efectividad de la evaluación formativa sobre el desarrollo de la motivación, la autonomía, el compromiso con la tarea y la superación personal de 14 estudiantes españoles; añadiendo además que los docentes al incorporar procesos de reflexión y mejora de su práctica consiguieron un mayor desarrollo profesional y mejora del clima en el aula. Por su parte Farinha \& Figueira (2016), basándose en un estudio mixto con el objetivo de exponer como los docentes conciben la evaluación formativa, arrojó que no existió relación entre la evaluación formativa frente a la práctica pedagógica; sin embargo, si admitieron que la evaluación formativa permitió controlar el proceso educativo. Por otro lado, la investigación de Rocha y De la Cruz (2018), obtuvo 
resultados difusos respecto de la propuesta de evaluación formativa la cual mostró algunas desventajas que disminuyeron con un trabajo planificado, una retroalimentación muy precisa y permanente para que los estudiantes alcancen los aprendizajes no logrados. Por su parte, Mejía (2012) afirmó que la evaluación formativa tiende al empleo de diversos recursos a fin de recuperar evidencias y valorar la acción observada, entre los cuales destacan el diseño, elaboración y aplicación de instrumentos diversificados; así como la comunicación empática de los resultados de tal forma que motiven y no desanimen, para lograr formar individuos competitivos.

Diversos trabajos de investigación desarrollados bajo el enfoque cualitativo, del tipo investigación-acción, determinaron, hasta qué punto, las estrategias de evaluación formativa contribuyen al mejoramiento de los aprendizajes. Algunos de dichos trabajos, demostraron que los estudiantes participaron activamente en la reconstrucción de sus aprendizajes. La evaluación formativa permite identificar las fortalezas y debilidades de la práctica pedagógica de los docentes, favorece la adquisición del conocimiento al propiciar la mejora del proceso de aprendizaje de los estudiantes, quienes reciben del docente respuesta a sus inquietudes mediante la retroalimentación, ya que permite la creación de aprendizajes significativos, permitiendo que los estudiantes mejoran sus aprendizajes y los docentes su práctica (Ortega, Santamaría y Gil, 2017; Cubillos, León y Rodríguez, 2018).

La investigación cualitativa de tipo etnográfica para delinear un proceso de evaluación formativa a partir de las actividades evaluativas que realizan los docentes en el aula, desarrollada por Pasek y Mejía (2017), se basó en la observación directa y el diario de campo como instrumentos. Al respecto lograron determinar que los docentes, al ofrecer una retroalimentación inmediata en el desarrollo mismo de la clase, realizaron una evaluación formativa, pero de una manera intuitiva y la mayoría de docentes $(87,5 \%)$ realizó la exploración monitorearon a los estudiantes durante las actividades de aula; pero, no todos resaltaron logros, ni promovieron la autoevaluación y la coevaluación; aun así, se determinó que el proceso fue útil para desarrollar una evaluación formativa consciente y sistemática. Por otro lado, Bizarro, Sucari y Quispe (2019), desarrollaron una investigación documental de tipo cualitativo con el objetivo de responder a las siguientes preguntas: qué y cómo se entiende la evaluación formativa, qué, para qué, con qué, cuándo, cómo se evalúa, y quienes evalúan. Sus resultados concluyeron que se debe valorar el desempeño individual y colectivo de los estudiantes que coparticipan en su evaluación, retroalimentándose en función a las evidencias, generando de esta manera, una transformación de la práctica evaluativa en la mejora de los aprendizajes con autonomía y reflexión.

Sobre el modelo de evaluación debe ser formativo, López (2012) consideró que debe ser continuo e integrado al currículo y sus resultados deben servir para mejorar al mismo currículo y los procesos que permiten aprender a los estudiantes. Por su parte Pérez, Enrique, Carbó y González (2017) consideraron la evaluación formativa como un proceso que constata y valora, permitiendo tomar decisiones que optimizan el proceso de enseñanza-aprendizaje, buscando ser humanizador más que calificador; Dicha concepción coincide en varios puntos con lo vertido por Chaviano, Baldomir, Coca y Gutiérrez (2016); Moreno (2012); Hamodi, López y López (2015) y López (2012). Discrepando en alguna medida con los autores citados, Ibarra y Rodríguez (2007) y Tejada (2011) muestran que tanto la evaluación formativa y la calificativa tienen aspectos comunes que las acercan tales como la validez (correspondencia entre lo que debería evaluarse y lo que se evalúa), confiabilidad (instrumento de medición cuantitativa o cualitativa de un mismo atributo), impacto educativo (estímulo y la retroalimentación), aceptabilidad (por estudiantes y maestros), costos (en la elaboración de las pruebas). Por otra parte, Casanova (2012) consideró habitual que se confunda "evaluación formativa" con "evaluación continua", tomada esta última como una incesante toma de pruebas, exámenes por lo que debe considerarse como "calificación continua".

Publicaciones más actuales conciben la evaluación como un proceso a través de la práctica docente; tales como Hortigüela, Pérez \& Moreno (2018) quienes lo asumieron desde la importancia en la toma de decisiones con la intención de mejorar los aprendizajes de los estudiantes. De forma coincidente Salazar (2018) afirmó que se trata de un proceso sistemático, que acopia información, para permitirle al docente emitir un juicio de valor sobre los aprendizajes alcanzados debido a su 
participación en las actividades de enseñanza desarrolladas. La idea convergente acerca de la evaluación tiende a incrementar su relevancia dentro del proceso enseñanza-aprendizaje (Deneen y Brown, 2016; Alcaraz 2015, Mejía, 2012; Mora, 2004; y Escudero, 2003). La evaluación es producto de numerosos cambios en el tiempo. Al respecto Andrade (2019) desarrolló un estudio estableciendo tres períodos. El primero, de finales del siglo XX, evaluar era medir lo que para Deneen y Brown (2016) tenía poca utilidad formativa al no existir retroalimentación efectiva por cuanto la evaluación estaba centrada en la "evaluación de los aprendizajes" al evidenciar el rendimiento de los estudiantes, lo que servía para elaborar informes de interés público. El segundo periodo, de inicios del siglo XXI que considera el cambio en la concepción de la evaluación del aprendizaje hacia la evaluación para el aprendizaje, que de acuerdo con Deneen y Brown (2016) permite al estudiante aprender de los aciertos y errores; asimismo, Sanmartí (2008) afirmó que las dificultades del proceso llevan a comprender las causas y a buscar estrategias para superarlas. El tercer periodo, el actual, se caracteriza por el equilibrio entre la evaluación del aprendizaje y la evaluación para el aprendizaje, evidenciado por un lado en el manejo de las evaluaciones estandarizadas y de la información que ofrecen y por el otro, en el manejo de las intenciones de la evaluación y de la retroalimentación efectiva.

Los procedimientos evaluativos permiten recopilar evidencias de aprendizajes, involucrando técnicas y procedimientos cuya validez deberá garantizarse por el evaluador, lo que significa que evalúa los criterios e indicadores establecidos previamente, por lo que la evidencia que recojan deberá ser variada, suficiente y relevante (Moreno, 2012). Mientras que los procedimientos y técnicas hacen referencia a las formas de evaluar, los instrumentos evaluativos como una prueba escrita, una rúbrica o una lista de cotejo son los que recogen efectivamente las evidencias deben ser construidos. Por su parte Förster (2017) mencionó que, si bien debe diseñarse una tabla con los objetivos de aprendizaje junto con los indicadores y/o criterios de evaluación, se requiere habilidad cognitiva, tipo y cantidad de ítems adecuados, además de la ponderación del grupo de ítems por indicador de evaluación. Lo anterior evidencia que un adecuado desarrollo del procedimiento de evaluación requiere además del conocimiento docente, un compromiso ético y una elevada responsabilidad social.

En la realidad peruana, esta propuesta reconocida normativamente por el Minedu en los años 2012, 2016 y 2019, no ha sido impulsada lo suficiente para que su aplicación sea una realidad; hecho que al parecer ocurre en varios países de Latinoamérica como México, donde Monzón (2015) advirtió que dicha evaluación es reconocida como necesaria para mejorar la práctica docente, pero que no se traduce en una real aplicación, manteniéndose la brecha entre el "deber ser" y el "ser"; aspecto que conlleva a considerar que aún subyace la práctica tradicional en la función docente.

En las observaciones realizadas a los docentes, se evidenció desidia para incorporar la evaluación formativa a la práctica pedagógica más que falta de claridad conceptual acerca de la propuesta. Dicha desidia se generó al advertir que la nueva forma de evaluar toma más tiempo, requiere plantear preguntas problematizadoras, por lo cual deben desarrollar técnicas y habilidades que creen no poseer, llegando incluso al convencimiento que solo las adquirirían mediante capacitaciones.

Esta situación relatada, no mostraba que se estaba logrando el enfoque por competencias, ni se evidenciaba una buena evaluación formativa, pese a estar planteadas en el Currículo Nacional de la EBR. Se pudo observar que los docentes seguían, subrepticiamente, evaluando por contenidos y que la única motivación que tenían los estudiantes seguía siendo extrínseca, es decir buscar satisfacer con sus calificaciones a terceros. Por lo expuesto, la evaluación seguía siendo uno de los puntos más conflictivos de la propuesta curricular, en la cual se proponía pasar a una evaluación de carácter formativo cuando aún la tradicional les resultaba más cómoda a los docentes. En dicho contexto se consideró imprescindible evaluar la evaluación formativa desde el punto de vista del aporte, qué significa como estrategia para la mejora de los aprendizajes de los estudiantes, teniendo en cuenta que la evaluación debe ser enfocada en principio para el aprendizaje. En función de lo planteado se formuló la siguiente pregunta de investigación ¿qué aspectos impiden el despliegue de la evaluación formativa en la práctica docente, como estrategia para la mejora de los aprendizajes en los estudiantes del nivel primaria? 


\section{Metodología}

El presente trabajo se enmarca dentro del paradigma cualitativo-interpretativo. Se adaptó una metodología dialógica a fin de analizar creencias, valores, prejuicios y sentimientos de los entrevistados inmersos en el hecho a analizar a fin de producir conocimiento sobre un aspecto de la realidad educativa. Se ha desarrollado el diseño fenomenológico para recabar experiencias individuales subjetivas de un grupo de trabajo. La población estuvo conformada por 8 docentes del nivel primario de una institución educativa pública del distrito de San Martín de Porras, Lima. Respecto de los participantes la mayor parte (83\%) fueron del género femenino y la edad promedio fue de 45 años para los docentes participantes. Por considerarse una investigación cualitativa, los hechos se construyen desde la manifestación de los sujetos y del análisis de las acciones de los mismos, por lo que se asumió una postura de verificación de las interpretaciones (Lankshear y Knobel, 2000).

El trabajo se basó en el análisis de cuatro categorías, de acuerdo a los sugerido por Tenbrink (2006), las cuales fueron: a) Preparación del proceso de evaluación, que contuvo a su vez cinco subcategorías: especificar los juicios a emitir y las decisiones a tomar (o posibles decisiones a tomar), describir la información necesaria, localizar la información ya disponible, decidir cómo y cuándo conseguir la información necesaria y construir o seleccionar los instrumentos de recogida de información; b) Recojo de información; que contuvo dos subcategorías: obtener la información necesaria y analizar y registrar información; y finalmente, c) Retroalimentación en la práctica docente, que tuvo a su vez tres subcategorías a saber: formular juicios, tomar decisiones e implementar acciones y resumir y dar a conocer los resultados de la evaluación.

El proceso de desarrollo se llevó a cabo en cuatro momentos: primero, se recabó toda la información posible; segundo, la triangulación de la información en busca de comprender a profundidad el fenómeno en estudio; tercero, la construcción del conocimiento derivado de las experiencias, presentado en forma narrativa o descriptiva y en la que se adjuntará el nivel de reflexión expresado por los docentes; cuarto, descripción de los aspectos que interactúan con el caso en estudio, tales como históricos, culturales, económicos, políticos.

\begin{tabular}{|c|c|}
\hline 1 & ¿Qué entiende Ud. por Evaluación formativa? \\
\hline 2 & $\begin{array}{l}\text { ¿Qué importancia tendría preparar el proceso de la evaluación formativa para la gestión } \\
\text { del aprendizaje? }\end{array}$ \\
\hline 3 & $\begin{array}{l}\text { ¿Está a disposición de los docentes la información requerida para esta evaluación o cómo } \\
\text { deben obtenerla para poder planificar la evaluación formativa? }\end{array}$ \\
\hline 4 & $\begin{array}{l}\text { En la planificación del proceso ¿Los docentes deben tomar en cuenta las posibilidades } \\
\text { que tienen para recoger la información estableciendo formas, tiempos e instrumentos } \\
\text { para la evaluación formativa? }\end{array}$ \\
\hline 5 & $\begin{array}{l}\text { ¿Qué aspectos considera que son los que se interponen en el recojo adecuado de la } \\
\text { información que se requiere en la evaluación formativa? }\end{array}$ \\
\hline 6 & $\begin{array}{l}\text { ¿Los docentes se encuentran preparados para analizar la información recogida, así como } \\
\text { para registrar la información pertinente qué les permita una retroalimentación efectiva } \\
\text { en la evaluación formativa? }\end{array}$ \\
\hline 7 & ¿Qué procesos considera que requerirá la retroalimentación para lograr los aprendizajes? \\
\hline 8 & $\begin{array}{l}\text { ¿Por qué aún se observan casos en que los resultados de la evaluación formativa no son } \\
\text { empleados en la adecuación del proceso enseñanza aprendizaje? }\end{array}$ \\
\hline 9 & $\begin{array}{l}\text { ¿Qué aspectos determinan que los docentes realicen o no la retroalimentación requerida } \\
\text { para cerrar el proceso de evaluación formativa? }\end{array}$ \\
\hline
\end{tabular}

Figura 1. Cuestionario de entrevista a expertos.

$1 \quad$ ¿Considera necesaria la evaluación formativa en la EBR?, ¿Por qué? 


\begin{tabular}{|c|l|}
\hline 2 & ¿Cuál es la razón de ser de los criterios y objetivos de evaluación? \\
\hline 3 & $\begin{array}{l}\text { ¿La evaluación debe planificarse con anticipación o puede desarrollarse en algún } \\
\text { momento del proceso educativo? }\end{array}$ \\
\hline 4 & ¿Qué información tiene para planificar sus evaluaciones? \\
\hline 5 & Si no tiene la información necesaria, ¿cómo la ubica? \\
\hline 6 & ¿Es conveniente evaluar imprevistamente? \\
\hline 7 & $\begin{array}{l}\text { ¿Cuál es para usted la mejor forma de evaluar a sus estudiantes y qué debe usar para } \\
\text { ello? }\end{array}$ \\
\hline 8 & Concluida la evaluación de sus estudiantes, ¿Qué hace con sus resultados? \\
\hline 9 & ¿Cuáles son las fases de la evaluación formativa?, ¿Cuál es el objetivo de cada fase? \\
\hline 10 & ¿La evaluación formativa es sumativa o continua? \\
\hline
\end{tabular}

Figura 2. Cuestionario para entrevista a docentes.

Por ser un estudio de casos (Stake, 1994) dentro de una investigación participativa tiende a ser multimodal, por lo que se consideró emplear la observación del proceso de evaluación directamente en el ejercicio de la práctica docente, el análisis de documentos tales como el plan de clase, las rúbricas de evaluación, grabación de las sesiones, entre otros; agregándose a estas técnicas las entrevistas semiestructuradas. De acuerdo con las técnicas mencionadas, se emplearán instrumentos como ficha de observación, ficha de registro de datos y cuestionario para las entrevistas (figuras 1 y 2).

De acuerdo con Stake (1994) en la organización de un estudio de casos múltiples se consideró los siguientes procesos: primero, selección del tema, que para el caso son los aspectos que afectan el adecuado uso de la evaluación formativa en una escuela de gestión estatal durante el año 2020 por parte de los docentes del nivel primario; segundo, fue la triangulación de la información, buscando comprender a profundidad el fenómeno en estudio; tercero, fue la construcción del conocimiento derivado de las experiencias, que se presentaron en forma narrativa o descriptiva y en la que se adjuntó el nivel de reflexión expresado por los mismos docentes haciendo referencia a las diferentes categorías presentes en el contexto y; cuarto, con el propósito de ubicar en el contexto al lector se describieron los aspectos que interactúan con el caso en estudio (históricos, culturales, económicos, políticos, entre otros).

\section{Resultados}

Acerca de los propósitos del proceso de evaluación, la revisión de las programaciones de los docentes permitió conocer que en varios casos no se proponían criterios de evaluación (sujetos: S1, S2, S6); otros proponían criterios no coherentes con los logros de aprendizaje (S3, S4, S5) y en algunos casos si utilizaron criterios pertinentes al logro de aprendizaje (S7, S8). Solamente en uno de los casos observados se registró la formulación de objetivos de evaluación pertinentes a los logros de aprendizaje (S8); por otro lado, mayormente se planteaban objetivos de evaluación que no guardan relación con el aprendizaje esperado (S2, S3, S4, S5, S7) o no se planteaban (S1, S6).

Con respecto a la planificación del proceso de evaluación las observaciones permitieron conocer que mayormente en la planificación de la evaluación los docentes no consideraban la información de las necesidades de aprendizajes; solo en dos de los casos observados (S7 y S8) se estableció que la información requerida estaba relacionada con las evidencias de aprendizaje. Si bien se detectó que algunos docentes ubicaban en los documentos pertinentes las competencias y capacidades de aprendizaje previstas, éstas no guardaban relación con las evidencias y los estándares de aprendizaje, en consecuencia, no es posible considerar que identifican la información en función al propósito de aprendizaje. Esto podría deberse al hecho de no tener claro la información que podría recogerse durante el desarrollo de la sesión, pues se observó que no se recoge la información adecuada y pertinente. 
En la mayoría de casos no tenían registrada ni la forma ni el tiempo de recojo de información sobre el logro de aprendizaje en sus planificaciones. Dos docentes establecieron las formas de evaluación en su planificación, así como el tiempo en que se recogería la información. Sólo una docente (S8) establecía en la ficha de observación los criterios de evaluación a diferencia de las demás docentes que empleaban la ficha de observación o la lista de cotejo, con ítems dicotómicos predominantemente y sin advertir los criterios de evaluación.

En cuanto a la aplicación de instrumentos en el proceso de evaluación se estableció que al margen del instrumento empleado los docentes no recogían la información que se necesitaba para un análisis pertinente. Más aún algunos no recogían ni la información del logro de aprendizaje (S6)

Acerca del procesamiento de información recogida se puso en evidencia que los docentes no ejecutaban el análisis de la información recogida en relación al logro de los aprendizajes y que la mayoría de los docentes observados no empleaba algún sistema de registro de información a partir del cual se pueda analizar el proceso. En general se observó que utilizan un registro limitado y solo dirigido a evidenciar el cumplimiento de las actividades.

Respecto a la meta evaluación se halló que la evaluación de los resultados consiste en verificar si se alcanzó el logro previsto (S1, S2, S4, S5); asimismo las preguntas que realizan algunos no guardan relación con el propósito del aprendizaje. (S6, S7, S8). Los docentes observados en la mayoría de casos no presentaban los resultados de la evaluación, limitándose a comentar a los estudiantes si están bien o sugerir que se haga mejor en el futuro, más aún en un caso se observó que se presentaron los resultados en forma selectiva.

Acerca del Feedback las observaciones realizadas a todos los sujetos de la investigación permitió detectar que ninguno de ellos realizaba acción alguna que indicara decisión para desarrollar y/o mejorar el proceso educativo a través de la retroalimentación, evidenciándose en los docentes al conocer los resultados de una evaluación que mientra algunos se limitaron a recomendar no cometer errores por cuanto afecta sus calificaciones ( $\mathrm{S} 1, \mathrm{~S} 2, \mathrm{~S} 3, \mathrm{~S} 5)$, otros no realizaron ninguna acción dirigida a reforzar y/o corregir el proceso educativo. (S4, S6, S7 y S8).

Al indagar sobre la necesidad de la evaluación formativa en la EBR los docentes la consideraron necesaria por qué "ayuda al estudiante a mejorar su proceso de aprendizaje" (S1) y por qué "Aplicando la retroalimentación como un proceso efectivo para reflexionar acerca de lo logrado" (S1, S2, S3, S5, S6, S7, S8), así como que permite proponer un reto docente y mejorar las dificultades de enseñanza aprendizaje buscando nuevas estrategias de enseñanza" (S4, S7, S8) y sobre la razón de ser de los criterios y objetivos de evaluación las respuestas fueron imprecisas al referirse a los criterios "Conocer lo que el estudiante va a lograr en sus aprendizajes" (S1), "se debe tener en cuenta los criterios para calificar a los estudiantes" (S3, S6, S8), "La razón principal de los criterios es valorar el propósito de lo que se quiere enseñar" (S7), "los criterios de evaluación nos permiten identificar si el desarrollo de las actividades se encuentra orientado"(S4),"Los criterios ... tienen como objetivo explicar de forma clara y detallada los aspectos que son evaluados" (S8). Asimismo, en sus comentarios se evidenció que buscaban evitar dar respuesta al propósito de emplear objetivos en la evaluación.

Por otro lado al preguntar si la evaluación debe planificarse con anticipación o puede desarrollarse en algún momento la mayoría de las docentes asumieron que la evaluación debe ser planificada pero argumentaron diferentes razones, para algunos "permitiría contar con los instrumentos y seleccionar los criterios" (S2, S7), otros consideraron que se debe "...tener en cuenta el momento de realizarla y no desordenar las actividades de aprendizaje de la sesión"(S3, S6), un docente argumentó que "La evaluación debe ser planificada con anticipación... y permite una retroalimentación para la mejora de los aprendizajes"(S4). Dos de las docentes respondieron que "Pueden ser ambas posibilidades... podemos planificar o aplicarla durante el desarrollo de la clase" (S1, S8) coludiéndose que podría ser imprevista. 
Acerca de la Información que dispone para planificar sus evaluaciones las respuestas halladas fueron variadas desde la información sobre las competencias y el avance o logro de los estudiantes ( $\mathrm{S}$, S2, S3, S6), pasando por fichas de textos y videos (S4) hasta información sobre instrumentos, competencias y desempeños estándar de acuerdo con el propósito. Asimismo, sobre la ubicación de la información necesaria, la respuesta unánime fue el currículo nacional y en un caso se mencionó la página web Aprendo en casa (S4)

Acerca de la conveniencia de evaluar imprevistamente seis de los ocho docentes opinaron que no lo era "por cuanto se necesita seleccionar los instrumentos de evaluación" (S2); "sería improvisar y no es útil" (S3, S4, S5); porque "debe evaluarse lo planificado" (S6, S7). Los dos que consideraron que si argumentaron que "dependía de la situación" (S1) y que "en la evaluación formativa la información se obtiene en cualquier momento de actividades quizá no programadas" (S8)

Al preguntar por la mejor forma de evaluar y qué debe usarse se obtuvo como repuesta la evaluación formativa, por auto, hetero o coevaluación $(\mathrm{S} 2, \mathrm{~S} 8)$ y el seguimiento para ayudar a que se siga aprendiendo (S4), otra propuesta fue la observación directa (S6) considerando como instrumentos: fichas de trabajo, exposiciones, conclusiones, por decir sus portafolios, utilizando listas de cotejo y escalas de observación, rúbricas (S6, S7, S8). Destaca, asimismo, que un docente respondiese que la mejor forma de evaluar era a través de la retroalimentación (S1) y en otro caso el planteamiento del uso de pruebas objetivas o de desarrollo como instrumentos. (S3) y al preguntarles qué hacen con los resultados de la evaluación, varios de ellos coincidieron en que a partir de ellos se busca nuevas estrategias de mejora. Se retroalimenta (S4, S5, S6, S7, S8). En otros casos indicaron que se les compara con los resultados del inicio y a partir de ello se planifica (S1), puesto que indican que debe reforzarse (S2) y se procede a repasar los aspectos aún no logrados (S3).

Acerca de las fases de la evaluación formativa y sus objetivos se halló que era un aspecto poco conocido para los docentes, los cuales se referían más a los momentos en que debe evaluarse, es así que manifestaron que las "fases son la inicial, la formativa que permite observar los procesos del desarrollo de sus competencias y la sumativa ayudan a ver si los estudiantes" (S2), agregando en otros casos la diagnóstica (S4, S5) o a la retroalimentación (S8). Finalmente coincidieron en todos los casos en que la evaluación formativa es continua, aunque sin mayor argumento.

\section{Discusión}

En base al análisis de las observaciones y de las respuestas recogidas, se llegó a conocer que en pocos casos las docentes proponen juicios de evaluación acertados, la mayoría no propone o no guardan relación con los logros esperados, como lógica consecuencia se da la incapacidad manifiesta para la formulación de los objetivos; esto desde ya evidencia en gran medida falta de capacidad para aplicar los conocimientos disponibles sobre los procesos evaluativos en general y sobre los de la evaluación formativa en particular. La opinión de los cuatro expertos fue coincidente con estas apreciaciones al establecer que uno de los aspectos limitantes es el formativo, es decir la falta de conocimiento acerca del enfoque formativo de la evaluación, evidenciando deficiente comprensión y empoderamiento de su finalidad, además de insuficientes conocimientos y dominio de las competencias e incapacidad para el análisis de estándares y formulación de criterios de evaluación. Esta realidad contradice la opinión de Zepeda y Förster (2017) los que consideraron fundamental la evaluación en la planificación para el aprendizaje y el diseñar un conjunto de criterios para tomar decisiones asociadas al proceso de evaluación de aprendizaje de los estudiantes.

Resultó evidente que los docentes no tienen claro qué y para qué se evalúa, es decir aún no se ha logrado clarificar el propósito de evaluación en todos los docentes y menos aún el propósito de la evaluación formativa. No sorprendió por ello que en el proceso de evaluación del nivel primaria de esta IE no se usara información sobre las necesidades de aprendizaje en la planificación y si bien registraron las competencias y capacidades que deben desarrollarse, no se precisaba ni la forma de evaluar ni tampoco el momento de aplicación. Lo observado coincidió con el trabajo de Farinha y 
Figueira (2016) quienes no hallaron relación entre la forma de concebir este tipo de evaluación con la práctica pedagógica en los maestros.

Asimismo, la opinión de los expertos, sobre el punto en discusión, estableció lo emocional como un segundo aspecto, al considerar la persistencia en desarrollar la evaluación desde el enfoque tradicionalista, dirigiéndola a calificar, recurriendo a la aplicación de exámenes e instrumentos. Dicho aspecto estaría limitando un adecuado manejo de los instrumentos apropiados para formar en la evaluación, tal como lo sostuvo Mejía (2012) quien consideró que los diferentes recursos empleados buscan recuperar evidencias con las cuales valorar la acción siendo una de ellas el diseño, elaboración y aplicación de instrumentos diversificados.

Por otro lado, es la retroalimentación el proceso más significativo de la evaluación formativa; pero que, en el caso analizado, las docentes de la IE no contaban con información organizada para el análisis, lo que de acuerdo con la opinión de los expertos se debía a que su formación tradicional. La carencia de referentes teóricos, explicarían las dificultades para incorporar criterios de evaluación y consecuentemente para el análisis de evidencias de aprendizaje. A juicio de expertos, al no estar preparados para analizar la información recogida, emitir juicios de valor o valorar la evidencia y registrar la información de manera objetiva, se imponía la apreciación o interpretación personal; hecho que imposibilitaba desarrollar una retroalimentación efectiva. Dicho aspecto fue demostrado por García y López (2015) quienes hallaron que la evaluación formativa desarrolló en los estudiantes la motivación, autonomía y compromiso con la tarea, ello como respuesta a la mejora de la práctica como consecuencia de la actitud reflexiva en los docentes.

Sin duda en el grupo docente analizado, la evaluación de los resultados fue para establecer quienes lograban el logro de aprendizaje, sin analizar el cómo y sin identificar nudos críticos que les indicaran la necesidad de reformular y replantear procesos y más aún sin comunicar a los estudiantes los resultados, negándose la oportunidad a que éstos pudieran reconocer su fortalezas y deficiencias; representa lo mencionado la negación de que la meta evaluación fuese una fase planificada y programada dentro de la evaluación; quizá ello llevó a que no fuese considerada la mejora del logro de los aprendizajes a través de la retroalimentación del proceso educativo, y como consecuencia no tomaban ninguna acción en este sentido, limitándose a recomendar "no seguir cometiendo fallas" pero sin considerar el reaprender como una forma de evitarlas, sin tomar en cuenta que

Por su parte Amaranti (2010) planteó que la retroalimentación debe formar parte del análisis de los resultados de evaluación en función a los objetivos o al desarrollo de capacidades y competencias. Así mismo Rowe y Wood (2008) sostuvieron que su efectividad está en relación con los estilos de aprendizaje y el planteamiento de estrategias que incluyan fortalezas y debilidades de los estudiantes y con el hecho de ser constructivas y motivantes.

Por otro lado, los expertos basados en su experiencia en el monitoreo y el acompañamiento docente, afirmaron que la falta de conocimientos sobre el enfoque de evaluación por competencias, desencadena una serie de falencias que se inicia al no articular la planificación y la evaluación, prosigue con la incapacidad para recoger evidencias de aprendizaje y culmina en una retroalimentación carente de pertinencia. La no interiorización de las ventajas de esta evaluación determinará que muchos docentes no planifiquen y/o ejecuten adecuadamente los procesos pues siguen concibiendo a la evaluación solo como medición del aprendizaje. Por otro lado, cabe mencionar la coincidencia con Monzón (2015), quien manifestó que en los países de la región se reconoce esta evaluación por competencias como necesaria; sin embargo, dicho reconocimiento no se ha traducido en aplicaciones concretas. Al respecto, en el presente estudio algunos docentes afirmaron que la formativa era la mejor evaluación y mencionaron diversos instrumentos que al respecto son empleados, pero la observación hecha de su práctica advirtió que se limitaban a usar la lista de cotejo. Ello deja en evidencia que la evaluación formativa no es una realidad aún en la práctica de los docentes analizados, puesto que no aceptan la modificación de su práctica pedagógica, dejando entrever una predisposición por la evaluación sumativa, así como la idea de que el análisis de los resultados debe conllevar un reforzamiento a los estudiantes, sin considerar que el no logro de aprendizajes recae en una responsabilidad compartida entre docente y estudiantes. 


\section{Conclusiones}

El análisis de las observaciones y de las respuestas de maestros y de los especialistas que monitorean y acompañan a los docentes permitieron identificar en primer lugar que no se tiene suficientemente claro el propósito de mejora integral del proceso educativo, de la evaluación formativa, ni tampoco de su aporte a la enseñanza y el aprendizaje que favorece al docente y al estudiante. Asimismo, fue posible sustraer evidencias del desconocimiento no solo del propósito sino también del conjunto de procesos que deben desarrollarse evidenciándose en la dificultad manifiesta para precisar y discriminar conceptos básicos que cimientan la evaluación formativa, como consecuencia de una formación profesional tradicional. Otro aspecto trascendente, dada la realidad analizada, es que a los maestros no se les monitoreaba ni se les acompañaban en los procesos evaluativos, especialmente en la toma de decisiones oportunas para reajustar el proceso de EA.

A ello se agrega que no se recogían evidencias de aprendizaje lo que equivalía a que no contaban con información de los avances y dificultades en relación al desarrollo de competencias y que la experiencia en el monitoreo y el acompañamiento docente de los expertos llevó a considerar que es consecuencia de la falta de conocimientos sobre el enfoque de evaluación por competencias; el no articular la planificación y la evaluación desencadenó las deficiencias observadas: incapacidad para recoger evidencias de aprendizaje y retroalimentación no pertinente ni acorde a los propósitos de aprendizaje y los criterios de evaluación. Es, asimismo, necesario mencionar que al no interiorizar las ventajas de esta evaluación no se ejecutan adecuadamente los procesos en muchos casos por considerarlos innecesarios, pues toman aún la evaluación como medición del aprendizaje. Por otro lado, ante las presiones de los organismos descentralizados, se prioriza cumplir la planificación curricular y no la reprogramación de las competencias no desarrolladas, para las cuales los docentes aducen que requieren de tiempo extra y exige creatividad, e innovación para diseñar nuevas situaciones de aprendizaje.

Con respecto a la preparación del proceso de evaluación se evidenció que no queda claro para este grupo docente el propósito del proceso de la evaluación formativa y falencias en la planificación del mismo. Las opiniones vertidas al respecto han llevado a establecer que la falta de conocimiento del enfoque formativo de la evaluación se evidenció en una deficiente comprensión y empoderamiento de la finalidad de la evaluación formativa; por otro lado, la falta de conocimiento y dominio de las competencias llevó a la incapacidad de analizar estándares para poder formular criterios de evaluación, concluyéndose que los docentes analizados no tienen claro qué y para qué se evalúa.

En cuanto al recojo de la información, que involucra la aplicación de instrumentos y el procesamiento de la información, debemos considerarla como poco adecuada y se asume que el factor responsable en este caso es de naturaleza emocional, manifestado como resistencia al cambio, como persistencia en evaluar desde el enfoque tradicionalista, centrado en calificar, medir a través de pruebas, exámenes e instrumentos similares. Por otro lado, el análisis de los resultados consistió en determinar quienes lograron resultados satisfactorios sin comunicarlo a todos los interesados.

El análisis del proceso de la retroalimentación como parte de la práctica de los docentes llevó a verificar que este proceso fue poco efectivo como consecuencia de la predisposición existente por la evaluación sumativa; a ello se agrega, que la responsabilidad al no lograr los objetivos del aprendizaje recae directamente sobre los estudiantes y por tanto hay que reforzar los contenidos no aprendidos, sin interesarse por qué no se aprendió ni cómo aprenden, no considerando la responsabilidad compartida en el fracaso del aprendizaje, lo que sentenciando que los docentes no están preparados para asumir el desafío de recoger y procesar información valiosa y auténtica del estudiante, menos aún para aceptar modificar su práctica pedagógica. 
De las respuestas dadas por los docentes se dedujo que no tienen suficientemente claro que la evaluación formativa procura una mejora integral del proceso educativo, tanto para el docente y el estudiante, por lo que no debe considerarse que el logro alcanzado es solo indicador del aprendizaje. Lo expuesto no se logró apreciar en las respuestas de la mayoría de los docentes, evidenciando desconocimiento del propósito no solo de sus procesos sino también de la evaluación formativa en su conjunto, sumándose a ello la dificultad manifiesta para precisar y discriminar conceptos básicos en los que se cimienta la evaluación formativa. Como la reflexión y autocritica supone ajustar la planificación respondiendo a las necesidades o nudos críticos identificados en el proceso para tomar decisiones oportunas el no hacerlo indica que no se monitorea ni se da el acompañamiento adecuado por lo cual no se pueden tomar decisiones oportunas para reajustar el proceso de enseñanza aprendizaje, siendo otra razón el no recoger evidencias de aprendizaje más relevante que brinden información de los avances y dificultades en relación al desarrollo de competencias. En definitiva, los docentes mantienen el enfoque de la evaluación del aprendizaje sin evidenciarse tránsito hacia la evaluación para el aprendizaje.

\section{Referencias}

Alcaraz- Salarirche, N. (2015). Aproximación Histórica a la Evaluación Educativa: De la Generación de la Medición a la Generación Ecléctica. Revista Iberoamericana de Evaluación Educativa, 8(1), 11 -25. https://bit.ly/3sqXULU.

Amaranti-Pesce, M. (2010). Evaluación de la educación: Concepciones y prácticas de retroalimentación de los profesores de lenguaje y comunicación de primer año de educación media, investigación cualitativa con estudio de caso. Recuperado de: https://bit.ly/39vSg2B.

Andrade-Ruiz, L. (2019). Ideas previas sobre evaluación en estudiantes de pedagogía en educación general básica y del programa de formación pedagógica para licenciados. ProQuest Number: 28180520, 1-24. https://bit.ly/3nIAEpb.

Bizarro, W., Sucari, W., y Quispe-Coaquira, A. (2019). Evaluación formativa en el marco del enfoque por competencias. Revista Innova Educación, 1(3): 374-390. https://doi.org/10.35622/j.rie.2019.03.r001.

Casanova, M. A. (2012). El diseño curricular como factor de calidad educativa. REICE. Revista Iberoamericana sobre Calidad, Eficacia y Cambio en Educación, 10(4), 6-20. https://bit.ly/3nMLwlS.

Chaviano-Herrera, O., Baldomir-Mesa, T., Coca-Meneses, O., y Gutiérrez-Maydata, A. (2016). La evaluación del aprendizaje: nuevas tendencias y retos para el profesor. Edumecentro, 8(4), 191-205. https://bit.ly/39xgJ7R.

Cubillos-Ayala, D. H., León-Téllez, F. S., \& Rodríguez-Useche, J. J. (2018). La evaluación formativa bajo el marco de la enseñanza para la comprensión hacia el fortalecimiento del pensamiento numérico [tesis de maestría, Universidad de la Sabana, Colombia]. Repositorio institucional http://hdl.handle.net/10818/34950.

Deneen, C. C. y Brown, G. T. L. (2016). The impact of conceptions of assessment on assessment literacy in a teacher education program. Cogent Education, 3(1), 1-14. https://doi.org/10.1080/2331186X.2016.1225380.

Escudero-Escorza, T. (2003). Desde los tests hasta la investigación evaluativa actual. Un siglo, el XX, de intenso desarrollo de la evaluación en educación. RELIEVE, 9(1), 11-43. https://bit.ly/35L05R0.

Farinha, M., \& Figueira, C. (2016). Das conceções às práticas de avaliação formativa no $1 .^{\circ}$ ciclo do ensino básico (Portugal). Campo Abierto. Revista de Educación, 36(1), 95-107. https://bit.ly/39CGrI5. 
Förster, C. (2017). Alfabetización evaluativa de los docentes. El poder de la evaluación en el aula: Mejores decisiones para promover aprendizajes. Santiago: Ediciones UC.

García-Herranz., S., \& López-Pastor, V. M. (2015). Evaluación Formativa y Compartida en Educación Infantil. Revisión de una Experiencia Didáctica. Qualitative Research in Education, 4(3), 269-298. doi:10.17583/qre.2015.1269.

Hamodi, C., López-Pastor, V. M., \& López-Pastor, A. T. (2015). Medios, técnicas e instrumentos de evaluación formativa y compartida del aprendizaje en educación superior. Perfiles Educativos, 37(147), 146-161. https://bit.ly/3sdgEOO.

Hortigüela-Acalá, D., Pérez-Pueyo, Á., \& Moreno-Doña, A. (2018). La evaluación formativa y compartida en la formación inicial del profesorado. Estudios pedagógicos (Valdivia), 44(2), 7-8. http://dx.doi.org/10.4067/S0718-07052018000200007.

Ibarra-Sáiz, M. S. y Rodríguez-Gregorio, G. (2007). El trabajo colaborativo en las aulas universitarias: reflexiones desde la autoevaluación. Revista de Educación, (344), 355-375. https://bit.ly/3bDjpmU.

Lankshear, C. y Knobel, M. (2000) Problemas asociados con la metodología de la investigación cualitativa. Perfiles educativos 6, 22(87), 6-27. https://bit.ly/39zolkt.

López-Pastor, V. M. (2012). Evaluación formativa y compartida en la universidad: clarificación de conceptos y propuestas de intervención desde la Red Interuniversitaria de Evaluación Formativa. Psychology, Society \& Education, 4(1), 117-130. https://bit.ly/3ozNXK2.

Mejía-Pérez, O. (2012). De la evaluación tradicional a una nueva evaluación basada en competencias. Revista Electrónica Educare, 16(1), 27-46. https://doi.org/10.15359/ree.161.3 .

Monzón-Troncoso, M. Y. (2015). Evaluación del aprendizaje: un recorrido histórico y epistemológico. Revista de Ciencias de la Educación Academicus, 6(1), 12-21. https://bit.ly/39dazJI.

Moreno-Olivos, T. (2012). La evaluación de competencias en educación. Sinéctica, (39), 1-20. https://bit.ly/3bJk1Ht.

Ortega-Quevedo, V., Santamaría-Cardaba N., y Gil-Puente, C. (2017). La evaluación formativa en una secuencia de enseñanza aprendizaje de Ciencias Experimentales. Revista Infancia, Educación y Aprendizaje-IEYA, 3(2), 203-209. https://doi.org/10.22370/ieya.2017.3.2.724.

Pasek-de Pinto, E., y Mejía, M. T. (2017). Proceso general para la evaluación formativa del aprendizaje. Revista Iberoamericana de Evaluación Educativa, 10(1), 177-193. http://dx.doi.org/10.15366/riee2017.10.1.009.

Pérez-Pino, M., Enrique-Clavero, J. O., Carbó-Ayala, J. E., y González-Falcón, M. (2017). La evaluación formativa en el proceso enseñanza aprendizaje. Edumecentro, 9(3), 263-283. https://bit.ly/39hPyh1.

Rocha-Gonzáles., S. A., \& De la Cruz-Trejo, J. A. (2018). Evaluacióń formativa de los aprendizajes esperados en educación primaria: ventajas y desventajas. RECIE. Revista Electrónica Científica de Investigación Educativa, 4(1), 443-454. https://bit.ly/3s9qPEc.

Salazar-Ascensio, J. (2018). Evaluación de aprendizaje significativo y estilos de aprendizaje: Alcance, propuesta y desafíos en el aula. Tendencias pedagógicas, (31), 31-46. http://dx.doi.org/10.15366/tp2018.31.001.

Sanmartí, N. (2018). La finalidad principal de la evaluación es la regulación tanto de la enseñanza como del aprendizaje. 10 ideas clave. En Evaluar para Aprender. Barcelona: Editorial GRAÓ.

Stake, R.E. (1994). Case studies. En N.K. Denzin y Y.S. Lincoln (Dirs.). Handbook of qualitative research, 236-247. London: Sage. 
Tejada-Fernández, J. T. (2011). La evaluación de las competencias en contextos no formales: dispositivos e instrumentos de evaluación. Revista de Educación, (354), 731-745. https://bit.ly/2LzA4g7.

Tenbrink, T. D. (2006). Evaluación: Guía práctica para profesores. New York, Nárcea. 